\title{
Resonance Effect of Binary Orbital Motion upon $\delta$ Scuti Type Stars
}

\author{
M. Paparó \\ Konkoly Observatory, Budapest P.O.Box. 67., Hungary
}

H. Shibahashi

Dept. of Astronomy, University of Tokyo, Tokyo 113-0033, Japan

C. Sterken

University of Brussels, Pleinlaan 2, 1050, Brussels, Belgium

\begin{abstract}
The colour behaviour of $\theta$ Tucanae with orbital phase is shown. A break in the residuals is checked as the criterion for stopping the period search. A resonance of the dominant pulsational mode and the period of ellipticity is reported, with a Resonance number of 72 .
\end{abstract}

\section{Ellipsoidal and reflection effect}

$\theta$ Tucanae, a $\delta$ Scuti type star, turned out to be a primary in a binary system similar to Algol (Paparó et al., 1996; Sterken, 1997; De Mey et al., 1998). The mass ratio (0.0896) is normal but the components are comparable in luminosity. The complexity of the binary system and pulsation are described by 13 frequencies. Two of them are connected to the binary nature, the period ratio is near
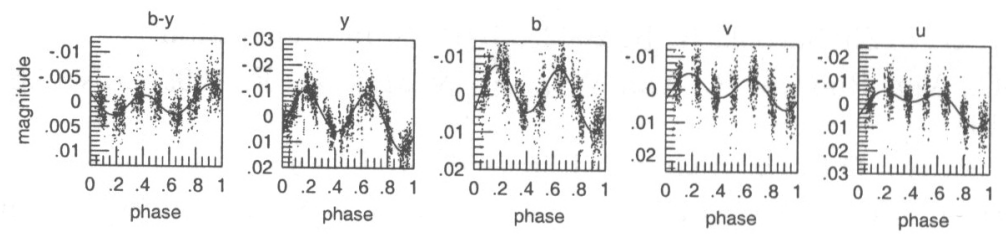

Figure 1. Colour behaviour according to the orbital phase. Mirror image and alternative deep and shallow minima are seen.

1:2. The shorter period $\left(0.2815 \mathrm{c} \mathrm{d}^{-1}\right)$ is connected to the ellipsoidal shape of the secondary. A reflection effect occuring once each orbital period causes alternating deep and shallow minima. The orbital period $\left(0.14206 \mathrm{c} \mathrm{d}^{-1}\right)$ is almost as long as the longest orbital period (around 9 days) in normal Algol-type systems.

The colour behaviour with orbital phase is shown in Fig. 1. Separation of the two effects reveals that the amplitude of the ellipsoidal effect decreases to shorter wavelengths while that of the reflection effect increases. The variations in colour and colour index are mirror images of each other. 

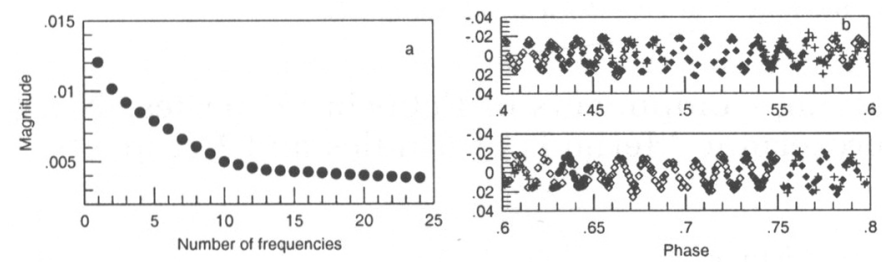

Figure 2. a. Residual depending on the number of frequencies prewhitened. b. The dominant period folded according to 3.552 day

\section{Where must we stop picking up frequencies ?}

In the colour behaviour figure the points show large scatter around the fitted curves, although the original data were prewhitened by all the pulsational frequencies published for $\theta$ Tucanae. However, this scatter is not larger than the residual of XX Pyx (Sterken, 2000). What is the reason for large residuals in well-studied cases?

An additional 11, and so altogether 24 frequencies, were picked up for $\theta$ Tucanae and the residuals after each step of prewhitening are plotted in Fig. $2 \mathrm{a}$. There is a definite break in the inclination. Before the break the modes have similar amplitude except the dominant mode. May we interpret the different inclination as modes excited with different amplitude level or the break in the residuals is the point where we are supposed to stop the period analyses?

\section{Resonance of orbital and pulsational periods}

Ten pulsational modes were tested for resonance with the characteristic times of the orbital motion. The dominant pulsation mode $\left(20.28 \mathrm{c} \mathrm{d}^{-1}\right)$ shows a definite resonance with the ellipsoidal effect. The resonance number is rather high (72), however, a similar value (76) was found for RZ Cas by Mkrtichian (these proceedings). The light curve of the dominant mode along the orbital phase is shown in Figure 2b. Different cycles are marked by different symbols. The present investigation is a first step for checking the tidal effect in Fourier parameters of excited modes. Details will be published elsewhere.

\section{References}

De Mey, K., Daems, K., \& Sterken, C. 1998, A\&A, 336, 527

Paparó, M., Sterken, C., Spoon, H.W.W., \& Birch, P.V. 1996, A\&A, 315, 400

Sterken, C. 1997, A\&A, 325, 5603

Sterken, C. 2000, in ASP Conf. Ser., Vol. 210, Delta Scuti and Related Stars, eds. M. Breger \& M. Montgomery (San Francisco: ASP), 99 